\title{
Safety during Transient Response in Direct Current Microgrids using Control Barrier Functions
}

\author{
K. C. Kosaraju, S. Sivaranjani, and V. Gupta
}

\begin{abstract}
We consider the problem of guaranteeing that the transient voltages and currents stay within prescribed bounds in Direct Current (DC) microgrids, when the controller does not have access to accurate system dynamics due to the load being unknown and/or time-varying. To achieve this, we propose an optimization based controller design using control barrier functions. We show that the proposed controller has a decentralized structure and is robust with respect to the uncertainty in the precise values of the system parameters, such as the load.
\end{abstract}

\section{INTRODUCTION}

The design and operation of microgrids, which are interconnected clusters of Distributed Generation Units (DGUs), loads and energy storage units interacting with each other through distribution lines, has been widely studied in the literature. This paper focuses on Direct Current (DC) microgrids [1] -[4]. The main control objective in DC microgrids is to ensure that the load voltage is stabilized to a desired value [5]. To achieve this objective, several control methods have been proposed, e.g., droop control [6], plug-and-play control [7], sliding mode control [8], passivity-based control [9], output regulation [10] and input-to-state stability based control [11].

However, there is limited literature studying the problem of maintaining the voltages and currents in the system within some prescribed bounds during transient operation in DC microgrids. Violating such constraints could lead to deterioration of equipment, ultimately leading to its failure. In this paper, we consider this problem. There are at least two challenges here. First, the control strategy should be decentralized (or at least distributed), for reasons of scalability and robustness. Second, since the knowledge of system parameters (such as the load values) is always imperfect, the controller should not rely on precise values of such parameters being available. As a first step towards solving the more general problem, we design a decentralized control algorithm for voltage and control regulation during transient operation for an islanded DC microgrid with purely resistive lines that does not require accurate information of load values.

Our controller design relies on casting the problem as one of safety and utilizing Control Barrier Functions (CBFs) to ensure that the system trajectories remain in a desired safe set. CBFs are now a widely accepted tool for designing safety based controllers [12]-[14]. CBFs guarantee the existence of control inputs under which a super-level set of a function (typically

K.C. Kosaraju, V. Gupta are with the Department of Electrical Engineering, University of Notre Dame, Notre Dame, IN 46556, USA (email: \{kkosaraj, vgupta2\}@nd.edu). S. Sivaranjani is with the Department of Electrical and Computer Engineering, Texas A\&M University, College Station, TX 77843, USA (email: sivaranjani@tamu.edu). representing a specification such as safety) is forward invariant under a given dynamics. However, they do not seem to have been widely explored in the context of DC microgrids. Our main contribution over the existing literature is that we utilize CBFs to design an optimization based controller for DC microgrids that guarantees constraint satisfaction during transient response, while being decentralized and not requiring a precise knowledge of the system parameters.

The paper is organized as follows. In Section II] we present the model of the DC microgrid and provide the problem formulation. In Section III], we propose a new control design using control barrier functions that solves the problem. In Section IV] we corroborate the proposed control design in simulations using a DC microgrid with four buck converters. Finally, in Section V] we conclude and provide some directions for future work. For completeness, a short review of control barrier functions is provided in Appendix A.

Notation: $\mathbb{R}^{n}$ denotes the space of $n$-dimensional real vectors and $\mathbb{R}$ denotes the space of real numbers. $\mathbb{1}$ denotes the vector of all ones with the dimension clear from the context. For a vector $v \in \mathbb{R}^{n}, v^{T}$ denotes its transpose, $\|v\|_{2}$ denotes its 2-norm, $v_{i}$ denotes its $i$-th element, and $[v]$ denotes a diagonal matrix with $v_{i}$ as the $i^{t h}$ diagonal entry, $\forall i \in\{1, \ldots, n\}$. For two vectors $u, v \in \mathbb{R}^{n}$, the inequality $u \leq v$ is interpreted element wise. A graph $\mathcal{G}=(\mathcal{V}, \mathcal{E})$ is defined by a node set $\mathcal{V}$ and an edge set $\mathcal{E}$. For a set $\mathcal{S},|\mathcal{S}|$ denotes its cardinality. A function $h: \mathbb{R}^{n} \rightarrow \mathbb{R}$ is said to be of class $C^{k}$ if the first $k$ derivatives exist and are continuous. A continuous function $h:[0, a) \rightarrow[0, \infty), a>0$ is said to belong to class $\mathcal{K}$ function if it is strictly increasing and $h(0)=0$. It is said to belong to class $\mathcal{K}_{\infty}$ if $a=\infty$ and $h(r) \rightarrow \infty$ as $r \rightarrow \infty$. Given $f(x): \mathbb{R}^{n} \rightarrow \mathbb{R}^{n}$ and $h(x) \in C^{1}, L_{f} h(x)$ denotes the Lie derivative of $h(x)$ along the direction of $f(x)$. A function $f: A \rightarrow B$ is Lipschitz if there exists a constant $L$ satisfying $\|f(b)-f(a)\|_{2} \leq L\|b-a\|_{2}$, for all $a, b \in A$ and class $C^{1}$ if it is continuously differentiable.

\section{DC Microgrid Model and Problem Formulation}

In this section, we introduce the DC microgrid model and formulate the problem of guaranteeing bounds on voltage and current during transient operation.

\section{A. DC microgrid model}

We model a DC microgrid by a connected undirected graph $\mathcal{G}=(\mathcal{V}, \mathcal{E})$, where each node $i \in \mathcal{V}$ represents a distributed generating unit (DGU) containing a voltage source, buck converter to step-down the voltage, and a load, and each edge $e \in \mathcal{E}$ represents a transmission line interconnecting the 


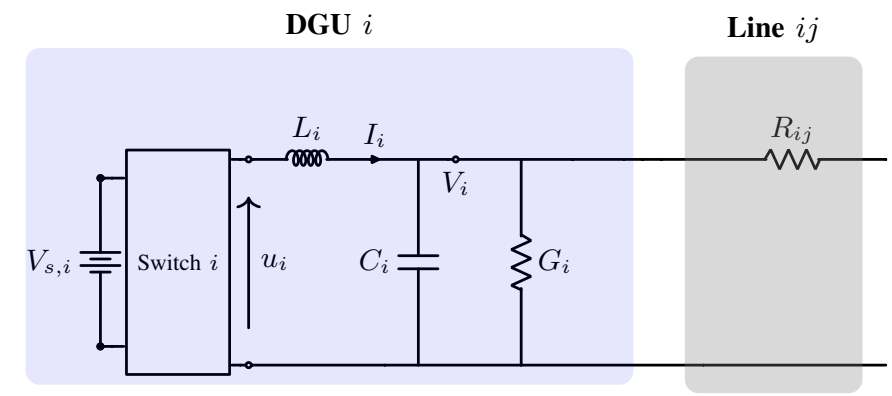

Fig. 1: Electrical scheme of DGU $i \in \mathcal{V}$ and line $k \sim\{i, j\} \in$ $\mathcal{E}, j \in \mathcal{N}_{i}$, where $\mathcal{N}_{i}$ is the set of the DGUs connected to DGU $i$.

corresponding DGUs. Let $|\mathcal{V}|=n$ and $|\mathcal{E}|=m$. For each edge $(u, v) \in \mathcal{E}$, arbitrarily choose one of the ends and assign it the value +1 ; similarly, assign the value -1 to the other end. Define the incidence matrix of the graph $\mathcal{G}$ by $\mathcal{B} \in \mathbb{R}^{n \times m}$ through the relation:

$$
\mathcal{B}_{i k}= \begin{cases}+1 & \text { if }(i, k) \in \mathcal{E} \text { and }+1 \text { was assigned to } i \\ -1 & \text { if }(i, k) \in \mathcal{E} \text { and }-1 \text { was assigned to } i \\ 0 & \text { otherwise }\end{cases}
$$

Note that, by construction,

$$
\mathcal{B}^{\top} 1=0 \text {. }
$$

We assume that each transmission line is purely resistive [15]. A schematic of each DGU is provided in Figure 1 and the variables used to specify the dynamic evolution of the microgrid are summarized in Table I

TABLE I: A summary of the used variables

\begin{tabular}{clcl}
\hline & States and input & & Parameters \\
\hline$I_{i}$ & Generated current & $L_{i}$ & Filter inductance \\
$V_{i}$ & Load voltage & $C_{i}$ & Shunt capacitor \\
$u_{i}$ & Control input & $G_{i}$ & Load impedance \\
& & $G_{l i}, G_{h i}$ & Bounds for load impedance \\
$V_{s, i}$ & Voltage source & $R_{t k}$ & Line resistance \\
\hline
\end{tabular}

The state of the DC microgrid is specified via the voltage at every node and current through every edge. At each node $i$, denote the current through the inductor $L_{i}$ by $I_{i}$, and denote the voltage across the load impedance $G_{i}$ by $V_{i}$. Stack these variables for all the nodes to form the current and voltage vectors $I, V: \mathbb{R}_{+} \rightarrow \mathbb{R}^{n}$. The control input at the $i$-th node is given by the duty-ratio of the buck converter of the $i$-th DGU, which is denoted by $u_{i} \in(0,1] . V_{s, i} \in \mathbb{R}_{+}$denotes the voltage source connected to the $i$-th node. Stack the control inputs and the voltage sources for all the nodes to define the control input and the voltage source for the entire system as $u: \mathbb{R}_{+} \rightarrow(0,1]^{n}$ and $V_{s} \in \mathbb{R}_{+}^{n}$, respectively. Finally, define positive definite and diagonal matrices $L, C, G \in \mathbb{R}^{n \times n}$ and $R_{t} \in \mathbb{R}^{m \times m}$ obtained by stacking and diagonalizing the resulting vector of DGU inductances, capacitances, load impedances and line resistances.
With these quantities defined, the dynamics governing the DC microgrid are given by the relations

$$
\begin{aligned}
-L \dot{I} & =V-V_{s} u \\
C \dot{V} & =I-G V-\mathcal{B} R_{t}^{-1} \mathcal{B}^{\top} V .
\end{aligned}
$$

For future reference, denote

$$
G_{p} \triangleq G+\mathcal{B} R_{t}^{-1} \mathcal{B}^{\top}
$$

We make the following assumptions in the paper.

Assumption 1. The current $I_{i}$ and the voltage $V_{i}$ are available by direct measurements at each $D G U i \in \mathcal{V}$.

Assumption 2. While the exact value of the load $G$ is unknown, upper and lower bounds are known as

$$
G_{l} \leq G \leq G_{h}
$$

where $G_{l}, G_{h}>0$ and the inequalities are interpreted elementwise.

\section{B. Problem formulation}

The control objective in the microgrid defined by the equations (2) is to design the control input $u$ such that the voltage $V$ across the load $G$ is stabilized to a desired value. In our formulation, we have assumed that the exact value of the load $G$ is unknown. Thus, the traditional objective of ensuring a specified value for the voltage may be too stringent. Instead, we assume that each load has a safe operating region in terms of a permitted lower bound $v_{l} \in \mathbb{R}$ and upper bound $v_{h} \in \mathbb{R}$ for the voltage across the load $G_{i}$. Consequently, we define the first control objective as follows:

Objective 1 (Safe voltage regulation). The voltage across the load $G$ must satisfy

$$
v_{l} \mathbb{1} \leq V(t) \leq v_{h} \mathbb{1}, \quad t \geq 0 .
$$

The second objective is to prevent the over or under drawing the current from the source. Thus, we define the second control objective as follows:

Objective 2 (Safe current regulation). The current through the load must satisfy the bounds

$$
I_{l} \mathbb{1} \leq I(t) \leq I_{h} \mathbb{1}, \quad t \geq 0,
$$

where $I_{l}, I_{h} \in \mathbb{R}$ are lower and upper bounds for the allowable values of the current.

The problem we consider can now be stated as follows.

Problem Statement: Given the DC microgrid as described in (2), we would like to design decentralized control inputs $u^{i}$ at each node $i \in \mathcal{V}$ to achieve Objectives 1 and 2, with each $u^{i}$ being computed using only local information about the state variables $I^{i}, V^{i}$ at node $i$.

Since the desired voltage across the load should be less than the supply voltage $V_{s}$, a trivial bound for $v_{h}$ in (5) is given by the source vector $V_{s}$. To see this more formally, note that for a given constant input $\bar{u} \in \mathbb{R}^{n}$, the corresponding steady state solution $(\bar{I}, \bar{V})$ of (2) satisfies

$$
\begin{aligned}
-\bar{V}+V_{s} \bar{u} & =0, \\
\bar{I}-G \bar{V}-\mathcal{B} R_{t}^{-1} \mathcal{B}^{\top} \bar{V} & =0 .
\end{aligned}
$$


Thus, the set of all feasible forced equilibria are given by the tuples $(\bar{I}, \bar{V}, \bar{u})$ that satisfy (7) and 8 Since $0<\bar{u} \leq$ 1 , the equation (7) implies that $\bar{V} \leq V_{s}$. We formalize this through the following assumption needed for the feasibility of the problem.

Assumption 3. We assume that

$$
v_{l} \leq v_{h}<V_{s}
$$

Finally, we assume that the problem is feasible.

Assumption 4. The problem stated above is feasible in the sense that there exists at least one control sequence achieving Objectives 1 and 2. In particular, the initial conditions for the voltages $V$ and the currents $I$ are in the sets defined in (5) and (6).

\section{Proposed SOLUTION}

The problem formulated above poses two difficulties. The first is that of guaranteeing the safety objectives 1 and 2 . The second is the limited knowledge of the load $G$. For pedagogical ease, we tackle these issues sequentially and present our solution to the problem formulated above in three steps:

- We first show how Objective 1 can be guaranteed when the load $G$ is known.

- We then extend the solution to the case when only a lower and an upper bound on the load $G$ are known.

- Finally, we extend the solution to also include Objective 2 .

The proofs of all the results are provided in the Appendices.

\section{A. Guaranteeing Objective 1 with known load}

We begin by designing a controller that guarantees objective 1 when the load $G$ is known. We utilize Control Barrier Functions (CBFs) for the purpose. A brief introduction to CBFs is provided in Appendix A for the interested reader.

Specifically, for all nodes $i \in \mathcal{V}$, we propose the controller obtained by solving the following optimization problem:

$$
\begin{aligned}
u_{i, 1}^{\mathrm{opt}}= & \arg \min _{a_{i} \in \mathbb{R}}\left\|a_{i}\right\|^{2} \\
\text { s.t. } & a_{i} V_{s, i}-V_{i}+\eta_{l, i}\left(I_{i}-G v_{l} \mathbb{1}\right) \geq 0 \\
& -a_{i} V_{s, i}+V_{i}-\eta_{h, i}\left(I_{i}-G v_{h} \mathbb{1}\right) \geq 0 \\
& 0 \leq a_{i} \leq 1,
\end{aligned}
$$

where $0 \leq \eta_{l, i}, \eta_{h, i} \leq 0$ are tuning parameters.

We now show that this controller stabilizes the system to the safe set (1).

Theorem 1. Consider the problem formulated in Section II with the load $G$ being known. The controller $u_{i, 1}^{\text {opt }}$ designed in (10) ensures that the system (2) satisfies Objective 1 .

Proof. See Appendix B.

Note that the last constraint in the QP 10 models the physical limits of the control input.

Remark 1 (Decentralized controller). In the proposed optimization based controller, computing $u_{i, 1}^{\mathrm{opt}}$ requires only local information of the states $\left(V_{i}, I_{i}\right)$. Thus, the controller has a decentralized structure as required.
The proposed controller is independent of the exact values of the parameters $L$ and $C$. Thus, even if we have limited knowledge of these parameters, the proposed controller provides some robustness with respect to variation in the values of these parameters.

\section{B. Guaranteeing Objective 1 with unknown load}

While the decentralized controller presented in Proposition 1 ensures that the voltage $V(t)$ lies with in the prescribed safe set, it requires an accurate knowledge of the load $G$. In practice, the exact value of the load is unknown and usually tends to change slowly with time. We now extend the controller presented above to not require this knowledge and hence be robust with respect to the precise value of the load $G$.

Specifically, we consider the controller obtained by solving the following optimization problem:

$$
\begin{aligned}
u_{i, 2}^{\mathrm{opt}}= & \arg \min _{a_{i} \in \mathbb{R}}\left\|a_{i}\right\|^{2} \\
\text { s.t. } & a_{i} V_{s, i}-V_{i}+\eta_{l, i}\left(I_{i}-G_{l} v_{l} \mathbb{1}\right) \geq 0 \\
& -a_{i} V_{s, i}+V_{i}-\eta_{h, i}\left(I_{i}-G_{h} v_{h} \mathbb{1}\right) \geq 0 \\
& 0 \leq a_{i} \leq 1,
\end{aligned}
$$

where $0 \leq \eta_{l, i}, \eta_{h, i} \leq 0$ are tuning parameters. We can then state the following result.

Theorem 2. Consider the problem formulated in Section II with only the bounds $G_{l}$ and $G_{h}$ on the load $G$ being known. The controller $u_{i, 2}^{\mathrm{opt}}$ calculated as proposed in (11) ensures that the system (2) satisfies Objective 1.

Proof. See Appendix C.

\section{Guaranteeing both Objectives 1 and 2}

To satisfy Objective 2, we can proceed in a similar fashion as above. However, we note that the voltages and currents in each DGU are not independent, and hence, their constraints need to be studied jointly. To this end, we note that if we define

$$
\begin{gathered}
\tilde{I}_{l} \triangleq \max \left\{v_{l} G_{l} \mathbb{1}, I_{l}\right\} \\
\tilde{I}_{h} \triangleq \min \left\{v_{h} G_{h} \mathbb{1}, I_{h}\right\},
\end{gathered}
$$

where the operations max and min are defined elementwise, then satisfying the conditions $\tilde{I}_{l} \geq 0$ and $\tilde{I}_{h} \geq 0$ will ensure that both Objectives 1 and 2 are met.

We consider the controller obtained by solving the following optimization problem:

$$
\begin{aligned}
u_{i, 3}^{\mathrm{opt}}= & \arg \min _{a_{i} \in \mathbb{R}}\left\|a_{i}\right\|^{2} \\
\text { s.t. } & a_{i} V_{s, i}-V_{i}+\eta_{l, i}\left(I_{i}-\tilde{I}_{l, i} \mathbb{1}\right) \geq 0 \\
& -a_{i} V_{s, i}+V_{i}-\eta_{h, i}\left(I_{i}-\tilde{I}_{h, i}\right) \geq 0 \\
& 0 \leq a_{i} \leq 1,
\end{aligned}
$$

where $0 \leq \eta_{l, i}, \eta_{h, i} \leq 0$ are tuning parameters and state the following result.

Theorem 3. Consider the problem formulated in Section II The controller $u_{i, 3}^{\mathrm{opt}}$ calculated as proposed in (13) ensures that the system (2) satisfies both Objectives 1 and 2 
Proof. The proof is analogous to that of Theorems 1 and 2 and is omitted.

Remark 2. Although for notational and pedagogical ease, we have assumed in the above development that the lower bound $I_{l}$ and the upper bound $I_{h}$ for all the DGUs are the same, all the arguments above can be extended to consider heterogeneous bounds. In fact, in the simulation study below, we demonstrate the heterogeneous case.

\section{Simulation Results}

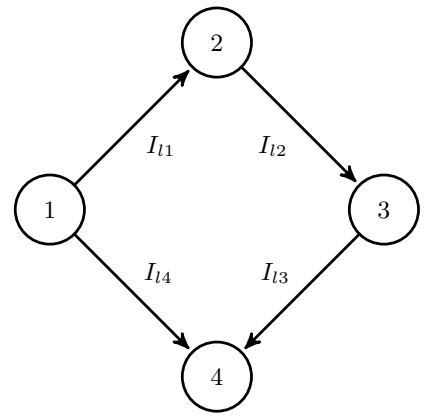

Fig. 2: DC microgrid considered with four buck converters.

In this section, we illustrate the proposed controller through a simulation study. We consider a Kron reduced DC microgrid consisting of four DGUs connected as shown in Figure 2. The parameters of the DGU and distribution lines are reported in Table II and Table III respectively. These parameters are similar to those used in [1], [9] and [16] for simulation and experimentation respectively. We assume that the desired nominal voltage for each DGU is $230.0 \mathrm{~V}$, with the safe region between $229.0 \mathrm{~V}$ and $231.0 \mathrm{~V}$. Moreover, the precise value of the load parameter $G$ is unknown and only the bounds $G_{l}=0.95 \times G$ and $G_{h}=1.05 \times G$ are known. Finally, for optimal working of the voltage source, we assume that the generating currents are bounded as shown in in Table II.

At time $t=0$, we start the system with a feasible voltage value. However, to test if our method can handle slight violations of the feasibility Assumption 4, the generating currents are initialized outside the prescribed safe region. Since the proposed controller assumes initialization within the feasible set, we use a numerical heuristic to bring the desired values quickly within the feasible region. Specifically, we modify the optimization problem (13) to

$$
\begin{aligned}
u_{i}^{\mathrm{opt}}= & \arg \min _{a_{i}, \epsilon_{l, i}, \epsilon_{h, i} \in \mathbb{R}} a_{i}^{2}+P_{l, i} \epsilon_{l, i}^{2}+P_{h, i} \epsilon_{h, i}^{2} \\
\text { s.t. } & a_{i} V_{s, i}-V_{i}+\eta_{l, i}\left(I_{i}-\tilde{I}_{l, i}\right)+\epsilon_{l, i} \geq 0 \\
& -a_{i} V_{s, i}+V_{i}-\eta_{h, i}\left(I_{i}-\tilde{I}_{h, i}\right)+\epsilon_{h, i} \geq 0 \\
& 0 \leq a_{i} \leq 1,
\end{aligned}
$$

for large values of constants $P_{l, i}$ and $P_{h, i}$. Once the currents and voltages enter the feasible set, we switch to the controller (13). The simulation is conducted for 0.5 sec. The tuning parameters of the controller are given in Table IV] After $0.25 \mathrm{sec}$ the load at all the nodes is increased by $5 \%$. The voltage and current signals of the resulting simulation are plotted in Figure 5 and Figure 4, respectively. The control input

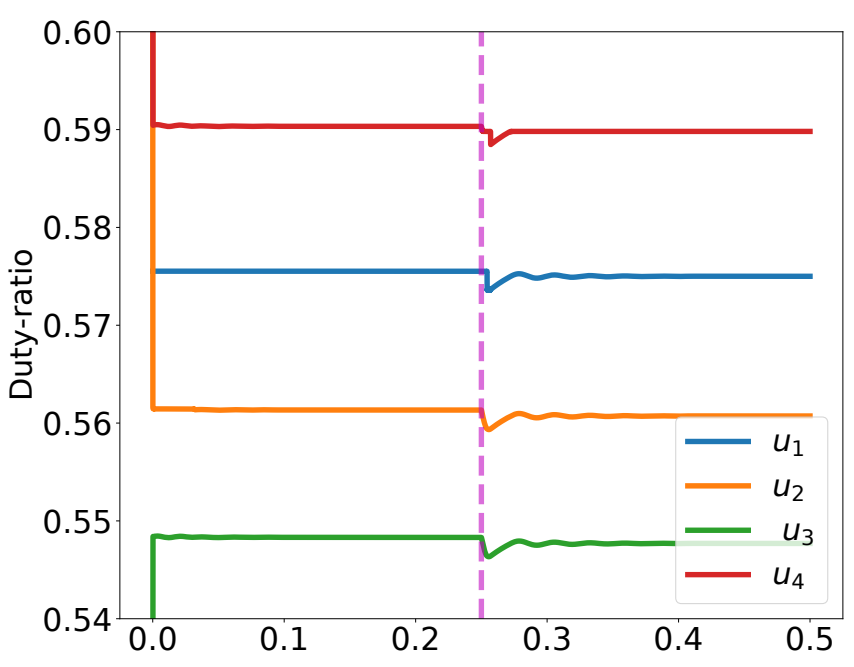

Fig. 3: Control input generated by the proposed controller.
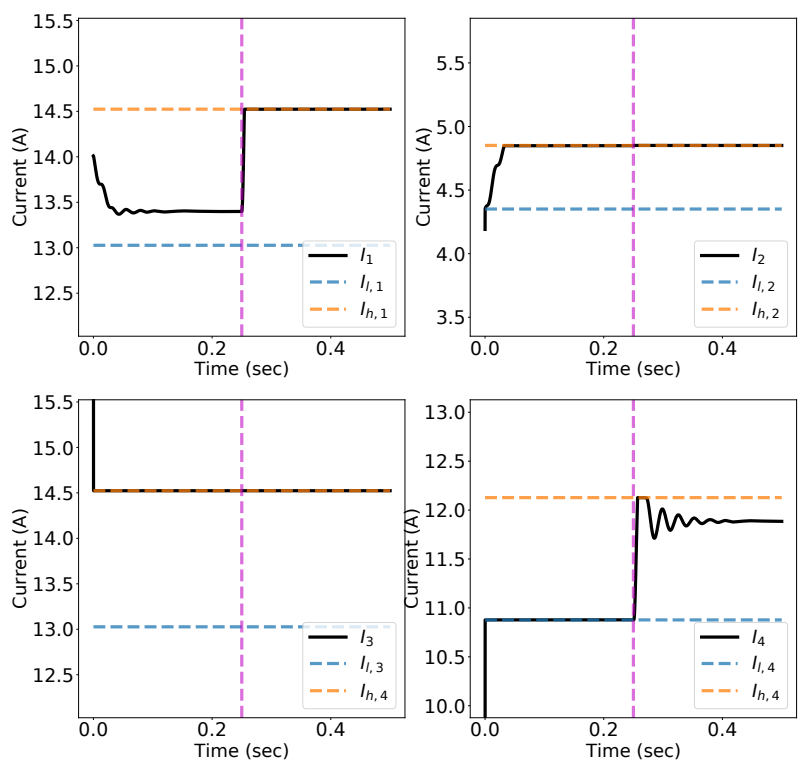

Fig. 4: Currents from the voltage source in each DGU.

generated by the optimization problem is plotted in Figure 3. We observe that the controller ensures satisfaction of both Objectives 1 and 2 (modulo the infeasbility of the specified initial condition) despite the abrupt load change.

\section{CONCLUSIONS AND FUTURE WORK}

We presented a new decentralized optimization based controller for DC microgrids. We used Control Barrier Functions to ensure that the voltage and current signals lie within the permitted safety bounds despite the load being unknown and potentially time-varying. Future research directions include extending this technique for ZIP loads and towards achieve safe current sharing in DC microgrids.

\section{REFERENCES}

[1] S. Trip, M. Cucuzzella, X. Cheng, and J. Scherpen, "Distributed averaging control for voltage regulation and current sharing in dc microgrids," IEEE Control Systems Letters, vol. 3, no. 1, pp. 174-179, 2018. 
TABLE II: Microgrid Parameters

\begin{tabular}{lc|cccc} 
DGU & & 1 & 2 & 3 & 4 \\
\hline$L_{i}$ & $(\mathrm{mH})$ & 1.8 & 2.0 & 3.0 & 2.2 \\
$C_{i}$ & $(\mathrm{mF})$ & 2.2 & 1.9 & 2.5 & 1.7 \\
$V_{i}^{\star}$ & $(\mathrm{V})$ & 230.0 & 230.0 & 230.0 & 230.0 \\
$V_{l, i}$ & $(\mathrm{~V})$ & 229.0 & 229.0 & 229.0 & 229.0 \\
$V_{h, i}$ & $(\mathrm{~V})$ & 231.0 & 231.0 & 231.0 & 231.0 \\
$G_{i}$ & $(\Omega)$ & $1 / 16.7$ & $1 / 50.0$ & $1 / 16.7$ & $1 / 20.0$ \\
$I_{l, i}$ & $(\mathrm{~A})$ & 13.0 & 4.4 & 13.0 & 11.0 \\
$I_{h, i}$ & $(\mathrm{~A})$ & 14.5 & 4.9 & 14.5 & 12.1
\end{tabular}

TABLE III: Line Parameters

\begin{tabular}{ll|cccc} 
Line & & 1 & 2 & 3 & 4 \\
\hline$R_{k}$ & $(\mathrm{~m} \Omega)$ & 70 & 50 & 80 & 60
\end{tabular}

TABLE IV: Controller Parameters

\begin{tabular}{l|cccc} 
node & 1 & 2 & 3 & 4 \\
\hline$\eta_{l, i}$ & 0.5 & 0.4 & 0.5 & 0.3 \\
$\eta_{h, i}$ & 0.4 & 0.3 & 0.5 & 0.4 \\
$P_{l, i}, P_{h, i}$ & $10^{23}$ & $10^{23}$ & $10^{23}$ & $10^{23}$
\end{tabular}

[2] M. S. Sadabadi, "Line-independent plug-and-play voltage stabilization and $l_{2}$ gain performance of de microgrids," IEEE Control Systems Letters, vol. 5, no. 5, pp. 1609-1614, 2020.

[3] K. Cavanagh, J. A. Belk, and K. Turitsyn, "Transient stability guarantees for ad hoc dc microgrids," IEEE Control Systems Letters, vol. 2, no. 1, pp. 139-144, 2017.

[4] J. J. Justo, F. Mwasilu, J. Lee, and J.-W. Jung, "AC-microgrids versus DC-microgrids with distributed energy resources: A review," Renewable and sustainable energy reviews, vol. 24, pp. 387-405, 2013.

[5] T. Dragičević, X. Lu, J. C. Vasquez, and J. M. Guerrero, "Dc microgrids-part i: A review of control strategies and stabilization techniques," IEEE Transactions on power electronics, vol. 31, no. 7, pp. 4876-4891, 2015.

[6] J. Zhao and F. Dörfler, "Distributed control and optimization in dc microgrids," Automatica, vol. 61, pp. 18-26, 2015.

[7] M. Tucci, S. Riverso, and G. Ferrari-Trecate, "Line-independent plugand-play controllers for voltage stabilization in dc microgrids," IEEE Transactions on Control Systems Technology, vol. 26, no. 3, pp. 1115$1123,2017$.

[8] M. Cucuzzella, R. Lazzari, S. Trip, S. Rosti, C. Sandroni, and A. Ferrara, "Sliding mode voltage control of boost converters in dc microgrids," Control Engineering Practice, vol. 73, pp. 161-170, 2018.

[9] K. C. Kosaraju, M. Cucuzzella, J. M. Scherpen, and R. Pasumarthy, "Differentiation and passivity for control of brayton-moser systems," IEEE Transactions on Automatic Control, 2020.

[10] A. Silani, M. Cucuzzella, J. M. A. Scherpen, and M. J. Yazdanpanah, "Output regulation for voltage control in dc networks with time-varying loads," IEEE Control Systems Letters, vol. 5, no. 3, pp. 797-802, 2021.

[11] A. Iovine, G. Damm, E. De Santis, M. D. Di Benedetto, L. GalaiDol, and P. Pepe, "Voltage stabilization in a dc microgrid by an isslike lyapunov function implementing droop control," in 2018 European Control Conference (ECC). IEEE, 2018, pp. 1130-1135.

[12] X. Xu, P. Tabuada, J. W. Grizzle, and A. D. Ames, "Robustness of control barrier functions for safety critical control," IFAC-PapersOnLine, vol. 48 , no. 27, pp. $54-61,2015$.

[13] A. D. Ames, S. Coogan, M. Egerstedt, G. Notomista, K. Sreenath, and P. Tabuada, "Control barrier functions: Theory and applications," in 2019 18th European Control Conference (ECC). IEEE, 2019, pp. 3420-3431.

[14] W. Xiao and C. Belta, "Control barrier functions for systems with high relative degree," in 2019 IEEE 58th Conference on Decision and Control (CDC). IEEE, 2019, pp. 474-479.

[15] R. H. Lasseter, J. H. Eto, B. Schenkman, J. Stevens, H. Vollkommer, D. Klapp, E. Linton, H. Hurtado, and J. Roy, "Certs microgrid laboratory test bed," IEEE Transactions on Power Delivery, vol. 26, no. 1, pp. 325332,2010

[16] S. Trip, R. Han, M. Cucuzzella, X. Cheng, J. Scherpen, and J. Guerrero, "Distributed averaging control for voltage regulation and current sharing in dc microgrids: Modelling and experimental validation," IFACPapersOnLine, vol. 51, no. 23, pp. 242 - 247, 2018, 7th IFAC Workshop on Distributed Estimation and Control in Networked Systems NECSYS 2018.
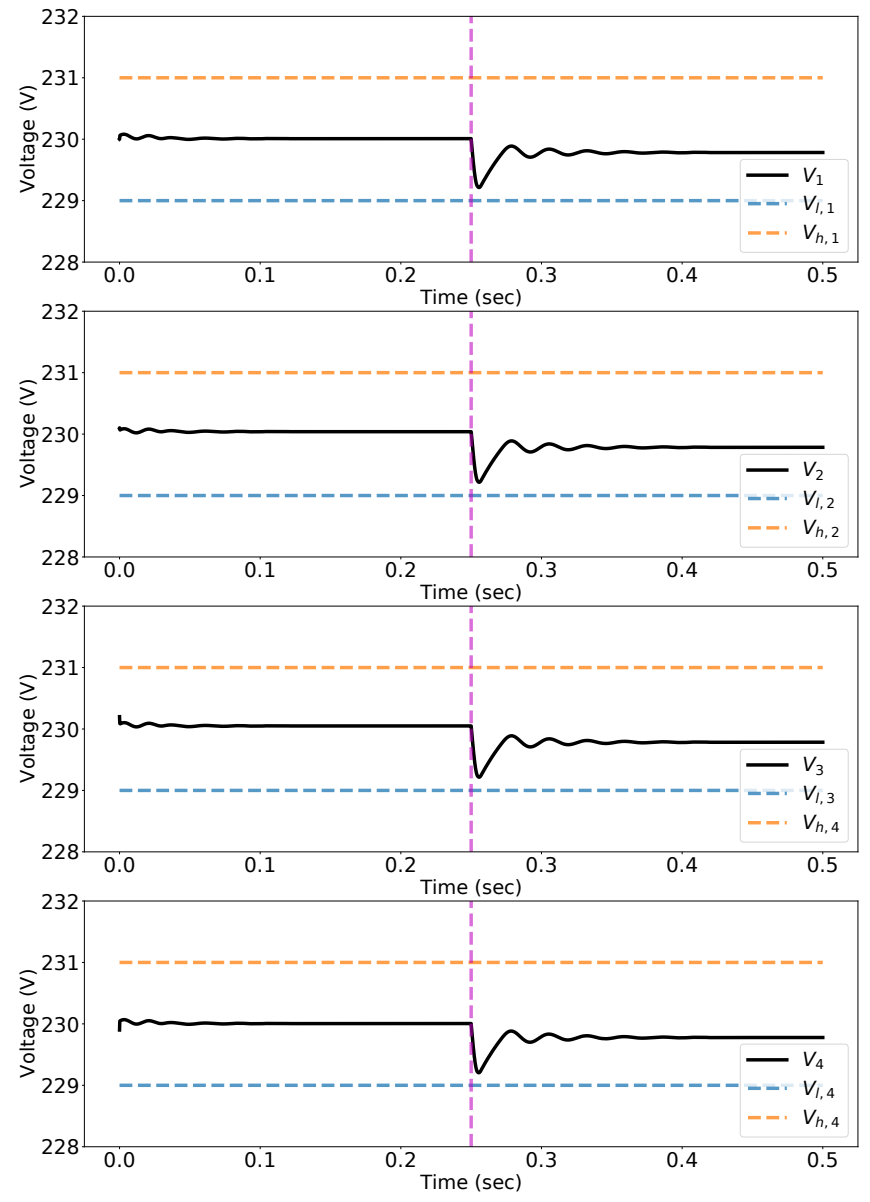

Fig. 5: Voltage across the load of each DGU, considering a load variation of $5 \%$ at time $t=0.25$ seconds.

\section{Vi. Appendix A: Control Barrier Functions}

Consider the affine nonlinear system

$$
\dot{x}=f(x)+g(x) u
$$

with $f$ and $g$ are locally Lipschitz continuous, $x \in \mathbb{R}^{n}$ and $u \in U \subset \mathbb{R}^{m}$. Let $\Omega$ be a zero super level-set of a continuously differentiable function $h: \mathbb{R}^{n} \rightarrow \mathbb{R}$, i.e.,

$$
\Omega=\left\{x \in \mathbb{R}^{n} \mid h(x) \geq 0\right\} .
$$

The function $h(x)$ is called a zeroing control barrier function (ZCBF), if there exists a locally Lipschitz extended class $\mathcal{K}$ function $\alpha$ such that

$$
\sup _{u \in \mathbb{R}^{m}}\left\{L_{f} h(x)+L_{g} h(x) u+\alpha(h(x))\right\} \geq 0 \quad \forall x \in \mathbb{R}^{n}
$$

where $L_{f} h(x)$ and $L_{g} h(x)$ denote the Lie derivative of $h(x)$ in the direction of $f$ and $g$, respectively. Given a ZCBF $h(x)$, define the set for all $x \in \Omega$

$$
K_{z c b f}(x)=\left\{u \in U: L_{f} h(x)+L_{g} h(x) u+\alpha(h(x)) \geq 0\right\} .
$$

Theorem 4 (See [12], [13]). Consider system (15], with $x \in$ $\mathbb{R}^{n}$ and $u \in U$ denoting the state and the input, respectively, $f$ and $g$ are locally Lipschitz. Let $\Omega$ be a zero super level-set of 
a continuously differentiable function $h(x)$, defined in (16). If the relative-degree of (15) w.r.t $h(x)$ is 1 (i.e., $L_{g} h(x) \neq 0$ ), then any Lipschitz continuous controller $u \in K_{z c b f}(x)$ renders the set $\Omega$ forward invariant and asymptotically stable.

For many physical systems, including the one considered in the paper, the relative degree $r$ is greater than one. In this case, as shown in [14], one can recursively define the control barrier functions $h^{i}(x)=L_{f} h^{i-1}(x)+\alpha^{I}\left(h^{i-1}(x)\right)$ and their zero super level sets $\Omega^{i}=\left\{x \in \mathbb{R}^{n} \mid h^{i-1}(x) \geq 0\right\}$, where $i \in\{1 \ldots r-1\}, \alpha^{1}, \cdots, \alpha^{r-1}$ are all class $\mathcal{K}$ functions and $h^{0}(x)=h(x)$. This is the construction used in the paper.

\section{Appendix B: Proof of Theorem 1}

Define the functions $b_{l}, b_{h}: \mathbb{R}^{2 n} \rightarrow \mathbb{R}^{n}$ as

$$
\begin{gathered}
b_{l} \triangleq V-v_{l} \mathbb{1} \\
b_{h} \triangleq v_{h} \mathbb{1}-V,
\end{gathered}
$$

and their corresponding zero super level-set of (19) as

$$
\Omega_{b} \triangleq\left\{(I, V) \in \mathbb{R}^{2 n} \mid b_{l} \geq 0, b_{h} \geq 0\right\} .
$$

Note that for all $(I, V) \in \Omega_{b}$, the voltage $V$ satisfies Objective 1 Since from Assumption 4, the initial condition is in this set, our proof below will show that the controller in $(10)$ renders the set $\Omega_{b}$ forward invariant and asymptotically stable for the system (2).

To this end, consider $b_{l}$ as the candidate zeroing control barrier function. By choosing $C^{-1} G_{p}$ as the class $\mathcal{K}$ function, the condition (17) for a zeroing control barrier function stated in the Appendix A simplifies to

$$
\dot{b_{l}}+C^{-1} G_{p} b_{l} \geq 0 .
$$

Multiplying $C$ on both the sides yields that (21) is equivalent to the condition

$$
C \dot{V}+G_{p}\left(V-v_{l} \mathbb{1}\right) \geq 0 .
$$

Using the relation $C \dot{V}+G_{p} V=I$ from (2) and 3), we can rewrite (22) as

$$
I-v_{l} G_{p} \mathbb{1} \geq 0 .
$$

Finally, since $\mathcal{B}^{\top} 1=0$, we can simplify 23 to the relation

$$
I-G v_{l} \mathbb{1} \geq 0 .
$$

Similarly, by considering $b_{h}$ as the zeroing control barrier function, we can obtain the condition

$$
-I+G v_{h} \mathbb{1} \geq 0 .
$$

Since the zero super level set of $b_{l}$ and $b_{h}$ is given by $\Omega_{b}$, Theorem 4 implies that if (24) and (25) hold, then Objective 1 is met.

However, there is no control input appearing in 24 and 25. This is a consequence of the fact that the relative degree of the system (2) with respect to either $b_{l}$ or $b_{h}$ is 2. Following [14], we can overcome this issue by recursively defining zeroing control barrier functions. To this end, define functions $B_{l}, B_{h}: \mathbb{R}^{2 n} \rightarrow \mathbb{R}^{n}$, given by

$$
\begin{aligned}
& B_{l} \triangleq I-v_{l} G \mathbb{1}, \\
& B_{h} \triangleq-I+v_{h} G \mathbb{1},
\end{aligned}
$$

with the zero super level set

$$
\Omega_{B} \triangleq\left\{(I, V) \in \mathbb{R}^{2 n} \mid B_{l} \geq 0, B_{h} \geq 0\right\} .
$$

To enforce (24), consider $B_{l}$ as the candidate zeroing control barrier function. By using $L^{-1}\left[\eta_{l}\right]$ as the class $\mathcal{K}$ function, we can write the condition in (17) as

$$
\dot{B}_{l}+L^{-1}\left[\eta_{l}\right] B_{1} \geq 0 \text {. }
$$

Multiplying both sides of 28 with $L$ and simplifying by using (2), we can rewrite this condition as

$$
-V+\left[V_{s}\right] u+\left[\eta_{l}\right]\left(I-G v_{l} \mathbb{1}\right) \geq 0 .
$$

Similarly, by considering $B_{h}$ as the candidate zeroing control barrier function, we can obtain the condition

$$
V-\left[V_{s}\right] u-\left[\eta_{h}\right]\left(I-G v_{h} \mathbb{1}\right) \geq 0 .
$$

Thus, we have shown that enforcing (29) and (30) ensures that the set $\Omega_{B}$ is forward invariant, which further enforces that $\Omega_{b}$ is forward invariant and asymptotically stable and hence that Objective 1 is met. But the first two constraints in the optimization problem (10) are equivalent to $(29)$ and $(30)$. Thus, we conclude that the controller $u_{i, 1}^{\mathrm{opt}}$ designed in 10 ensures that the system (2) satisfies Objective 1 .

\section{APPENDix C: Proof of TheOREM 2}

Following the arguments from the proof of Theorem 1, we know that Objective 1 is satisfied if $\Omega_{b}$ is rendered forward invariant. In turn, this can be ensured by a control input that yields $B_{l}, B_{h} \geq 0$. Since the exact value of $G$ is unknown, we impose the sufficient condition that

$$
\begin{aligned}
& I \geq v_{l} G \mathbb{1} \geq v_{l} G_{l} \mathbb{1}, \\
& I \leq v_{h} G \mathbb{1} \leq v_{h} G_{h} \mathbb{1} .
\end{aligned}
$$

In other words, define the two functions

$$
\begin{aligned}
& \hat{B}_{l} \triangleq I-v_{l} G_{l} \mathbb{1} \geq 0, \\
& \hat{B}_{h} \triangleq-I+v_{h} G_{h} \mathbb{1} \geq 0,
\end{aligned}
$$

with the super level set

$$
\hat{\Omega}_{B} \triangleq\left\{(I, V) \in \mathbb{R}^{2 n} \mid \hat{B}_{l} \geq 0, \hat{B}_{h} \geq 0\right\},
$$

which satisfies $\hat{\Omega}_{B} \subset \Omega_{B}$. We now consider $\hat{B}_{l}$ and $\hat{B}_{h}$ as the zeroing control barrier functions. Following the proof of Theorem 22, we can derive the conditions

$$
\begin{aligned}
-V+\left[V_{s}\right] u+\left[\eta_{l}\right]\left(I-G_{l} v_{l} \mathbb{1}\right) & \geq 0, \\
V-\left[V_{s}\right] u-\left[\eta_{h}\right]\left(I-G_{h} v_{h} \mathbb{1}\right) & \geq 0,
\end{aligned}
$$

in place of 295 and 30 The conditions 34 are equivalent to the first two constraints in the optimization problem (11). Thus, the controller $u_{i, 2}^{\mathrm{opt}}$ designed in (11) renders the set $\hat{\Omega}_{B} \subset \Omega_{B}$, and in turn, $\Omega_{b}$ forward invariant and asymptotically stable. Thus, the controller $u_{i, 2}^{\text {opt }}$ ensures that the system (2) satisfies Objective 1 in spite of lack of precise knowledge of the load $G$. 